\title{
Biodegradation of the xenobiotic organic disulphide 4,4'-dithiodibutyric acid by Rhodococcus erythropolis strain MI2 and comparison with the microbial utilization of $3,3^{\prime}$-dithiodipropionic acid and 3,3'-thiodipropionic acid
}

\author{
Jan Hendrik Wübbeler, Nadine Bruland, Milena Wozniczka \\ and Alexander Steinbüchel
}

Correspondence

Alexander Steinbüchel

steinbu@uni-muenster.de

Received 6 November 2009

Revised 29 November 2009

Accepted 2 December 2009
Institut für Molekulare Mikrobiologie und Biotechnologie, Westfälische Wilhelms-Universität Münster, 48149 Münster, Germany

Application of the non-toxic 3,3'-thiodipropionic acid (TDP) and 3,3'-dithiodipropionic acid (DTDP) as precursors for the microbial production of polythioesters (PTEs), a class of biologically persistent biopolymers containing sulphur in the backbone, was successfully established previously. However, synthesis of PTEs containing 4-mercaptobutyrate (4MB) as building blocks could not be achieved. The very harmful $4 \mathrm{MB}$ is not used as a PTE precursor or as the carbon source for growth by any known strain. As a promising alternative, the harmless oxidized disulfide of two molecules of $4 \mathrm{MB}, 4,4^{\prime}$-dithiodibutyric acid (DTDB), was employed for enrichments of bacterial strains capable of biodegradation. Investigation of novel precursor substrates for PTEs and comparison of respective strains growing on TDP, DTDP and DTDB as sole carbon source was accomplished. A broad variety of bacteria capable of using one of these organic sulphur compounds were isolated and compared. TDP and DTDP were degraded by several strains belonging to different genera, whereas all DTDB-utilizing strains were affiliated to the species Rhodococcus erythropolis. Transposon mutagenesis of $R$. erythropolis strain $\mathrm{MI} 2$ and screening of 7500 resulting mutants yielded three mutants exhibiting impaired growth on DTDB.

Physiological studies revealed production of volatile hydrogen sulphide and accumulation of significant amounts of $4 \mathrm{MB}, 4$-oxo-4-sulphanylbutanoic acid and succinic acid in the culture supernatants. Based on this knowledge, a putative pathway for degradation of DTDB was proposed: DTDB could be cleaved into two molecules of $4 \mathrm{MB}$, followed by an oxidation yielding 4-oxo-4-sulphanylbutanoic acid. A putative desulphydrase probably catalyses the abstraction of sulphur, thereby generating succinic acid and hydrogen sulphide.

\section{INTRODUCTION}

4,4'-Dithiodibutyric acid (DTDB), 3,3'-dithiodipropionic acid (DTDP) and 3,3'-thiodipropionic acid (TDP) are deployed in many different areas of research (Appel et al., 1995; Codognoto et al., 2007; Jang \& Keng, 2006; Kanayama \& Kitano, 2000; Saxena \& Gupta, 1984; Scott, 1968; Tsutsumi et al., 1998; Tuan \& Phillips, 1997). However, the biotechnological significance of these organic sulphur compounds (OSCs) is their application in studies for the improvement of polythioester (PTE) production (Lütke-Eversloh \& Steinbüchel, 2003). PTEs are micro-

\footnotetext{
Abbreviations: DTDB, 4,4'-dithiodibutyric acid; DTDP, 3,3'-dithiodipropionic acid; 4MB, 4-mercaptobutyric acid; 3MP, 3-mercaptopropionic acid; OSC, organic sulphur compound; PHA, polyhydroxyalkanoic acid; PTE, polythioester; 3SP, 3-sulphinopropionic acid; TBL, $\gamma$-thiobutyrolactone; TDP, 3,3'-thiodipropionic acid.
}

bially synthesized, non-biodegradable polymers containing sulphur in the thioester linkages of the backbone (Kim et al., 2005; Lütke-Eversloh et al., 2002a). They accumulate as hydrophobic inclusions in the cytoplasm of bacterial cells in a manner similar to polyhydroxyalkanoic acids (PHAs) (Lütke-Eversloh \& Steinbüchel, 2004). PHAs and PTEs are industrially and scientifically interesting materials, not only because they possess some unique characteristics regarding their biodegradability, microstructure, thermal behaviour and diverse mechanical properties, but also because many of them can be produced from nonpetrochemical resources (Steinbüchel, 2001).

Initially, PTE heteropolymers were detected in Ralstonia eutropha (Lütke-Eversloh et al., 2001a). This Gramnegative bacterium naturally accumulates PHAs at levels of up to more than $90 \%$ of the cell dry matter (Pedrós-Alio 
et al., 1985; Schlegel et al., 1961a), and it synthesizes various PTE heteropolymers, employing its unspecific PHA synthase if 3-mercaptopropionic acid (3MP), 3-mercaptobutyric acid (3MB), 3-mercaptovaleric acid (3MV), TDP or DTDP is provided as a precursor in addition to a second compound that serves as a carbon and energy source (Lütke-Eversloh \& Steinbüchel, 2003; Lütke-Eversloh et al., 2001a, b, 2002a). Later, PTE homopolymers consisting of $3 \mathrm{MP}, 3 \mathrm{MB}$ or $3 \mathrm{MV}$ were synthesized in a recombinant strain of Escherichia coli expressing a non-natural pathway (Lütke-Eversloh et al., 2002b; Thakor et al., 2005), if the respective OSC was provided as precursor. The use of DTDB or 4-mercaptobutyric acid (4MB) should yield PTE with $4 \mathrm{MB}$ building blocks possessing hitherto unknown and probably interesting properties. However, even though various strategies for biosynthesis have been applied, production of $4 \mathrm{MB}$-containing PTE has not yet been successful (Lütke-Eversloh \& Steinbüchel, 2003).

Although most of these precursor substrates are structural analogues of common cell metabolites (for a comparison see Fig. 1), it became obvious from previous studies (Bruland et al., 2009a, b; Wübbeler et al., 2006) that degradation was restricted to only few bacteria. Also, both established PTE-producing strains were not able to use any of the OSCs mentioned above as the sole carbon and energy source for growth. Furthermore, at present it is not possible to synthesize PTEs from simple carbon and inorganic sulphur sources. Thus, the establishment of microbial synthesis processes, independently of toxic and often expensive OSCs, by metabolic engineering of suitable micro-organisms is desirable. Prior to such a strain optimization, it was essential to obtain bacteria with the capacity to utilize precursors of PTE as the sole carbon source, to understand the catabolism of the respective OSC.

Extensive analyses have recently been accomplished to elucidate the catabolic pathways of TDP and DTDP in Variovorax paradoxus strain TBEA6 and Advenella mimigardefordensis strain $\mathrm{DPN}^{\mathrm{T}}$, respectively. Transposon mutagenesis employing the suicide plasmid technique described by Simon et al. (1983), as an established method to elucidate unknown metabolic pathways, can successfully be applied to both strains (Bruland et al. 2009a; Wübbeler et al. 2008). The putative pathway of microbial utilization of TDP with hitherto unknown and interesting enzyme reactions has recently been proposed (Bruland et al., 2009a). In the first step, the thioether is cleaved by a yet unidentified enzyme into 3-hydroxpropionate and 3MP. The latter is then sulphoxygenated by a novel cysteine dioxygenase homologue referred to as 3MP dioxygenase, yielding 3-sulphinopropionate (3SP). After linkage to coenzyme A (CoA) by a family III acyl-CoA transferase, the sulphur moiety is probably removed by a desulphinase, yielding propionyl-CoA, which is then further metabolized, most likely via the methylmalonyl-CoA pathway.

Catabolism of DTDP is initiated by a symmetrical cleavage of DTDP into two molecules of $3 \mathrm{MP}$ by a disulphide reductase (J. H. Wübbeler and others, unpublished results). As in TDP degradation (see above), a 3MP dioxygenase converts the sulphydryl group of $3 \mathrm{MP}$ into a sulphinic group, thereby yielding 3SP. A thiokinase then activates 3SP by ligating CoA with 3 SP to give 3SP-CoA (M. Schürmann and others, unpublished results). Subsequently, the necessary elimination of sulphite from $3 \mathrm{SP}-\mathrm{CoA}$ probably requires three enzymes catalysing yet unconfirmed conversions to yield propionyl-CoA, which is then further metabolized via the 2-methylcitric acid pathway (Wübbeler et al., 2008).

DTDB is evidently the oxidized form of two $4 \mathrm{MB}$ molecules (Fig. 1e). It is far less toxic and, in direct comparison with its symmetrical cleavage compound $4 \mathrm{MB}$, odourless, and also much cheaper. $4 \mathrm{MB}$ is hardly available as commercial product on the market. Therefore, it was of particular interest for the present study to gain preliminary insights into the metabolism of this OSC; especially important to investigate was the overall biodegradability of DTDB by cultivable micro-organisms and the occurrence of $4 \mathrm{MB}$ during degradation. Since it was predicted that $4 \mathrm{MB}$ is a valuable precursor substrate for the biotechnological production of novel PTEs, the elucidation of the hitherto unknown DTDB, and consequently $4 \mathrm{MB}$, catabolic pathways in bacteria would be helpful to improve

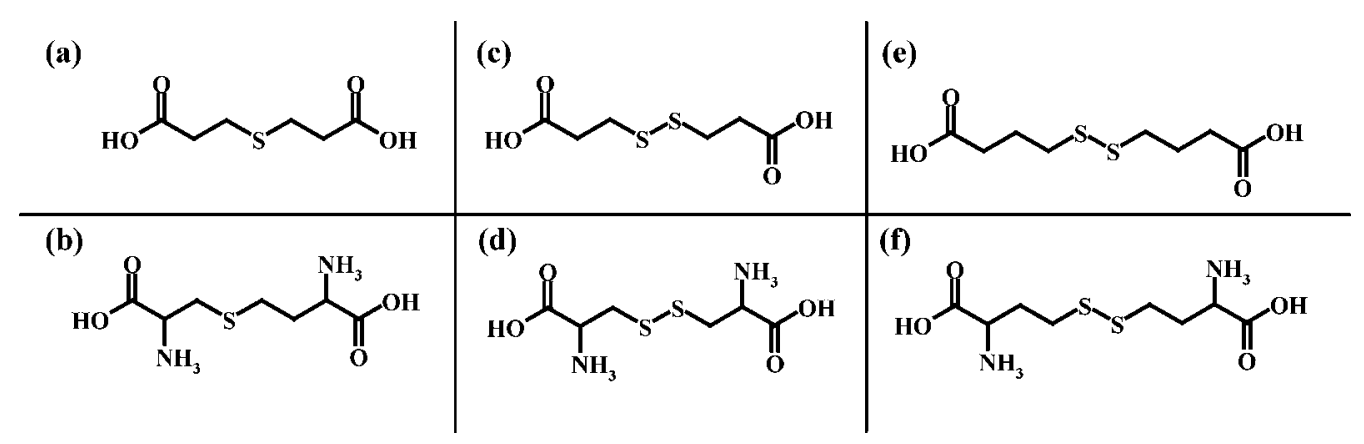

Fig. 1. Comparison of the chemical structures of the OSCs relevant to this study with those of the respective analogous cell intermediates. (a) TDP, (b) cystathionine, (c) DTDP, (d) cystine, (e) DTDB, (f) homocystine. 
and establish PTE synthesis to produce polymers containing $4 \mathrm{MB}$ building blocks. The use of DTDB as the precursor substrate could be advantageous to establish PTE production in bacterial strains showing a certain susceptibility to the toxic thiol $4 \mathrm{MB}$. Furthermore, a higher $4 \mathrm{MB}$ content in such a PTE might be achieved by heterologous expression of DTDB-cleaving enzymes in engineered recombinant strains.

\section{METHODS}

Sulphur-containing chemicals. DTDP and TDP were purchased from Acros Organics. DTDB was purchased from Sigma-Aldrich Chemie.

Growth conditions and storage of bacterial strains. All available bacterial strains investigated for their ability to utilize the PTE precursors as the sole source of carbon and energy are shown in Table 1, whereas the characterized natural isolates are listed in Table 2. Strains were maintained either on $0.8 \%(\mathrm{w} / \mathrm{v})$ nutrient broth (NB) and Luria-Bertani (LB) medium (Sambrook et al., 1989), or on mineral salts medium (MSM) (Schlegel et al., 1961b) at $30{ }^{\circ} \mathrm{C}$ containing the appropriate carbon source indicated in the text. Carbon sources were prepared as filter-sterilized $20 \%(\mathrm{w} / \mathrm{v})$ stock solutions and adjusted to $\mathrm{pH}$ 7.0. Solid media contained $1.8 \%(\mathrm{w} / \mathrm{v})$ purified agar-agar. Long-term storage was accomplished in screwcapped vials at $-70{ }^{\circ} \mathrm{C}$ as glycerol $(20 \%$, v/v) stocks or as lyophilizates.

Enrichment and isolation of mesophilic natural isolates capable of utilizing the respective OSC as the sole source of carbon and energy. Natural isolates catabolizing the OSCs investigated and compared in this study were obtained from $250 \mathrm{ml}$ flasks filled with $50 \mathrm{ml}$ sterile MSM (with an initial $\mathrm{pH}$ of 6.9) and $1 \%(\mathrm{w} / \mathrm{v}$ or $\mathrm{v} / \mathrm{v})$ of inoculum, which were taken from many different polluted locations. The OSCs were added as carbon source at concentrations between 0.2 and $1.0 \%(\mathrm{v} / \mathrm{v})$. The flasks were shaken for several days at $30{ }^{\circ} \mathrm{C}$ before aliquots were plated on solid MSM agar plates containing the respective OSC as the sole carbon source. After up to 7 days of incubation, colony material from these plates was repeatedly transferred to fresh plates until axenic cultures were achieved.

The verified available strains and isolates obtained, in association with their geographical origins and relevant phenotypes, are listed in Tables 1 and 2, respectively.

Biochemical characterization of natural isolates. Gram staining, the L-alanyl-aminopeptidase test, the potassium hydroxide test, as well as catalase and oxidase tests were performed according to Gerhardt et al. (1994). The susceptibility of the strains towards antibiotics was determined on complex media for the following antibiotics and concentrations: $150 \mu \mathrm{g} \mathrm{m}^{-1}$ ampicillin (Ap), $50 \mu \mathrm{g}$ $\mathrm{ml}^{-1}$ chloramphenicol $(\mathrm{Cm}), 10 \mu \mathrm{g} \mathrm{ml}{ }^{-1}$ gentamicin $(\mathrm{Gm}), 100 \mu \mathrm{g}$ $\mathrm{ml}^{-1}$ kanamycin $(\mathrm{Km}), 200 \mu \mathrm{g} \mathrm{ml} l^{-1}$ penicillin $(\mathrm{Pn}), 100 \mu \mathrm{g} \mathrm{ml}$ streptomycin $(\mathrm{Sm}), 12.5 \mu \mathrm{g} \mathrm{ml}^{-1}$ tetracycline $(\mathrm{Tc})$ and $25 \mu \mathrm{g} \mathrm{ml}^{-1}$ thiostrepton (Ts). API 20NE and 20E tests (bioMérieux) were performed to determine the assimilation of a variety of carbon sources and the presence of enzymes for taxonomic affiliation. The capacity to utilize different carbon sources, which is not included in the API tests, was investigated on MSM agar plates containing 0.1$1.0 \%(\mathrm{w} / \mathrm{v})$ of the respective carbon source.

Detection of inorganic sulphur compounds. Sulphate and sulphite contents in culture supernatants were determined with test strips (Merck), based on either a thorin-barium complex (sulphate detection) or a mixture of potassium hexacyanoferrate(II), zinc

Table 1. Bacterial strains from culture collections with proven inability to utilize DTDB, DTDP and TDP as the sole source of carbon and energy

Abbreviations: ATCC, American Type Culture Collection; DSM, Deutsche Sammlung von Mikroorganismen und Zellkulturen; ${ }^{\mathrm{T}}$, type strain

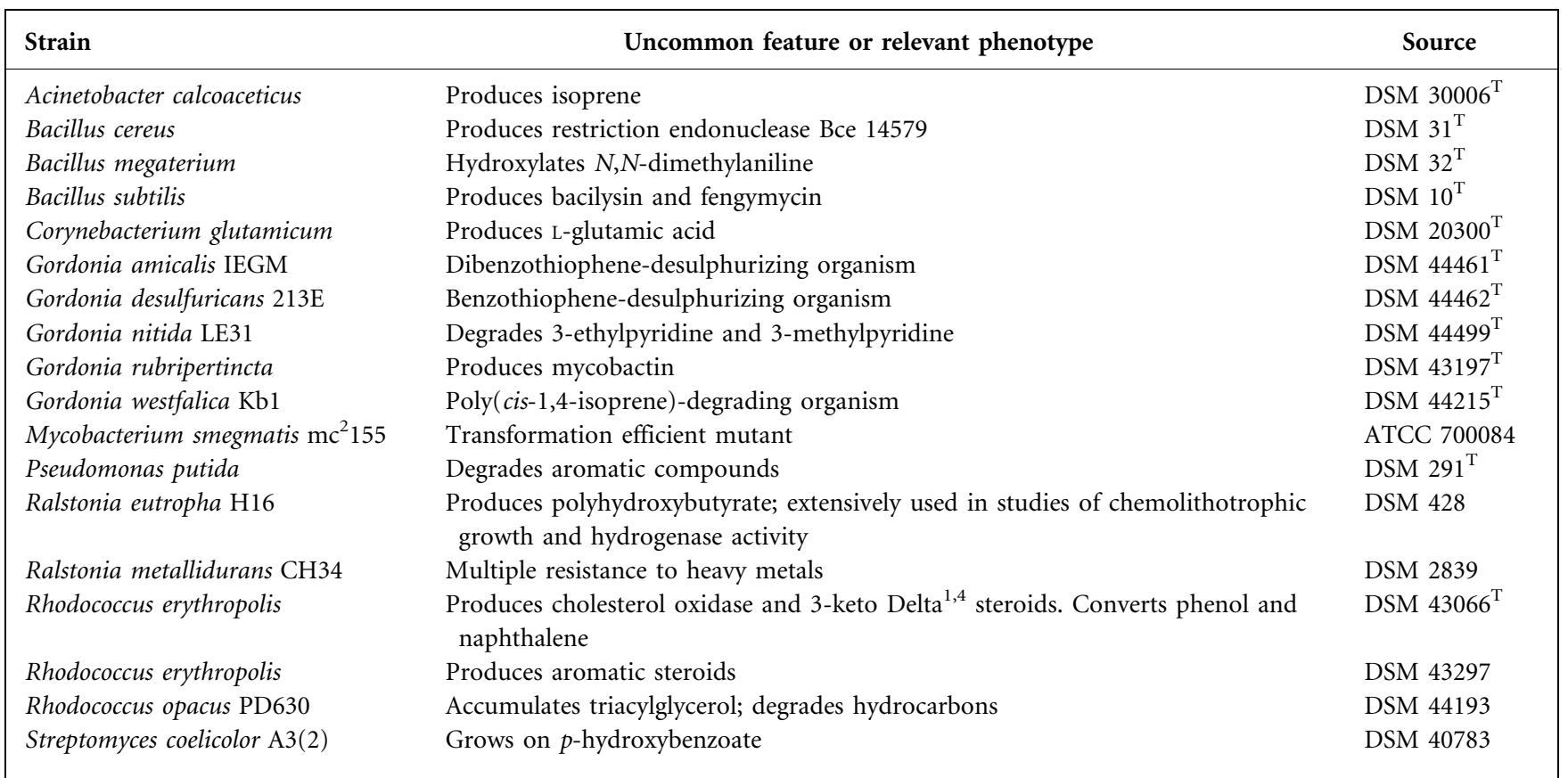


Table 2. Bacterial isolates from this and previous studies capable of using the indicated OSC as sole source of carbon and energy Abbreviations: Ap, ampicillin; Cm, chloramphenicol; Gm, gentamicin; Km, kanamycin; Pn, penicillin; Sm, streptomycin; Tc, tetracycline; Ts, thiostrepton.

\begin{tabular}{|c|c|c|c|c|}
\hline Strain designation & $\begin{array}{l}\text { OSC } \\
\text { utilized }\end{array}$ & Source of sample & $\begin{array}{l}\text { Susceptible to the } \\
\text { following antibiotics }\end{array}$ & Source or reference \\
\hline R. erythropolis DBR3 & DTDB & Matured compost & Sm, Ts & This study \\
\hline R. erythropolis DBR4 & DTDB & Matured compost & $\mathrm{Gm}, \mathrm{Sm}, \mathrm{Ts}$ & This study \\
\hline R. erythropolis DBRW & DTDB & Matured compost & $\mathrm{Gm}, \mathrm{Sm}, \mathrm{Ts}$ & This study \\
\hline R. erythropolis DBRQ & DTDB & Matured compost & Sm, Ts & This study \\
\hline R. erythropolis MI2 & DTDB & $\begin{array}{l}\text { Oil-contaminated material from } \\
\text { an absorber in a garage }\end{array}$ & $\mathrm{Cm}, \mathrm{Ts}$ & This study \\
\hline R. erythropolis E6 & DTDB & Soil sample of an inner courtyard & $\mathrm{Cm}, \mathrm{Ts}$ & This study \\
\hline R. erythropolis BEA1 & DTDP & $\begin{array}{l}\text { Soil sample in vicinity of a storage } \\
\text { site for waste thiochemicals }\end{array}$ & $\begin{array}{l}\text { Ap, Cm, Gm, Km, Pn, } \\
\text { Sm, Tc, Ts }\end{array}$ & This study \\
\hline R. erythropolis BEA2 & DTDP & $\begin{array}{l}\text { Soil sample in vicinity of a storage } \\
\text { site for waste thiochemicals }\end{array}$ & $\begin{array}{l}\text { Ap, Cm, Gm, Km, Pn, } \\
\text { Sm, Tc, Ts }\end{array}$ & This study \\
\hline R. erythropolis BEA3 & DTDP & $\begin{array}{l}\text { Soil sample in vicinity of a storage } \\
\text { site for waste thiochemicals }\end{array}$ & Ap, Cm, Gm, Pn, Sm, Tc, Ts & This study \\
\hline R. erythropolis BEA4 & DTDP & $\begin{array}{l}\text { Soil sample in vicinity of a storage } \\
\text { site for waste thiochemicals }\end{array}$ & $\begin{array}{l}\text { Ap, Cm, Gm, Km, Pn, Sm, } \\
\text { Tc, Ts }\end{array}$ & This study \\
\hline Cupriavidus taiwanensis BEA5c & DTDP & $\begin{array}{l}\text { Soil sample in vicinity of a storage } \\
\text { site for waste thiochemicals }\end{array}$ & Ap, Cm, Pn, Sm, Tc & This study \\
\hline Pseudomonas sp. strain MT-I1 & DTDP & Sewage sludge & $\mathrm{Gm}, \mathrm{Km}, \mathrm{Sm}$ & Toups et al. (2009) \\
\hline Bordetella sp. strain MT-I2 & DTDP & Sewage sludge & $\mathrm{Ap}, \mathrm{Cm}, \mathrm{Km}$ & Toups et al. (2009) \\
\hline Pseudomonas sp. strain MT-I3 & DTDP & Sewage sludge & $\mathrm{Cm}, \mathrm{Sm}$ & Toups et al. (2009) \\
\hline $\begin{array}{l}\text { Tetrathiobacter }(\text { Advenella }) \\
\text { mimigardefordensis }\end{array}$ & DTDP & $\begin{array}{l}\text { Matured compost after the } \\
\text { sieving process }\end{array}$ & Ap, Gm, Km, Pn, Sm, Tc & Wübbeler et al. (2006) \\
\hline Variovorax paradoxus SFWT & TDP & Sediments of a fresh water tank & $\mathrm{Cm}$ & Bruland et al. (2009a) \\
\hline Variovorax paradoxus TBEA6 & TDP & $\begin{array}{l}\text { Soil sample in vicinity of a } \\
\text { storage site for waste } \\
\text { thiochemicals }\end{array}$ & $\mathrm{Cm}, \mathrm{Km}, \mathrm{Tc}$, Ts & Bruland et al. (2009a) \\
\hline P. soli $\mathrm{TBEA} 3$ & TDP & $\begin{array}{l}\text { Soil sample in vicinity of a } \\
\text { storage site for waste } \\
\text { thiochemicals }\end{array}$ & $\mathrm{Cm}, \mathrm{Km}, \mathrm{Tc}, \mathrm{Ts}$ & Bruland et al. (2009a, b) \\
\hline Schlegelella thermodepolymerans & TDP & Activated sludge & $\mathrm{Ap}, \mathrm{Cm}, \mathrm{Km}, \mathrm{Sm}, \mathrm{Tc}$ & Elbanna et al. (2003) \\
\hline
\end{tabular}

sulphate and sodium nitroprusside (sulphite detection). Detection of volatile hydrogen sulphide production by micro-organisms was accomplished with lead acetate test strips (Fluka). Solubilized hydrogen sulphide was determined with the Visocolor Eco sulphide test kit (Macherey-Nagel) based on $N, N^{\prime}$-dimethyl-1,4-phenylene diamine, which is converted in two steps into methylene blue, if hydrogen sulphide is present. Application of the analytical test strips was performed according to the manufacturers' instructions.

Isolation, manipulation and transfer of DNA. Chromosomal DNA was isolated as described by Marmur (1961). Plasmid DNA was isolated by the method of Birnboim \& Doly (1979). Restriction enzymes and ligases were used according to the instructions of the manufacturers. Competent cells of E. coli were prepared by the $\mathrm{CaCl}_{2}$ procedure and transformed with $16 \mathrm{~S}$ rDNA ligated into plasmid pGEM-T Easy (Promega) (Hanahan, 1983).

Electroporation of Rhodococcus erythropolis strain MI2 was accomplished based on the methods described by Desomer et al. (1990) and Kalscheuer et al. (1999).

PCR. Amplifications of genomic DNA were accomplished according to the procedures described by Innis et al. (1990).
Sequencing of 16S rRNA genes. PCR amplifications of the $16 \mathrm{~S}$ rRNA genes were carried out using the oligonucleotides $27 f\left(5^{\prime}\right.$ GAGTTTGATCCTGGCTCAG-3') and 1525r (5'-AGAAAGGAGGTGATCCAGCC- $3^{\prime}$ ) as primers, which are complementary to conserved regions of the 16S rRNA gene of $E$. coli. The PCR products were purified using the NucleotrapCR extraction kit (Macherey-Nagel) and, if necessary, ligated into the cloning vector pGEM-T Easy (Promega) prior to sequencing.

The sequences of the 16S rRNA genes were determined by the chaintermination method (Sanger et al., 1977), applying a SequiTherm long-read cycle sequencing kit (Epicentre Biotechnologies) and IRD800-labelled oligonucleotides (MWG Biotech). Sequence reactions were accomplished by using a GeneReadIR 4200 DNA analyser (LI-COR). The following oligonucleotides were used as primers: $27 \mathrm{f}$, 343r (5'-CTGCTGCCTCCCGTA-3'), 357f (5' -TACGGGAGGCAGCAG-3'), 519r [5'-G(T/A)-ATTACCGCGGC(T/G)GCTG-3'], 536f [5'-CAGC(C/A)GCCGCGGTAAT(T/A)C-3'], 803f (5'-ATTAGATACCCTGGTAG-3'), 907r (5' -CCGTCAATTCATTTGAGTTT$\left.3^{\prime}\right)$, 1114f (5'-GCAACGAGCGCAACCC-3'), 1385r [5'-CGGTGTGT(A/G)CAAGGCCC-3'] and 1525r, as well as the M13 universal primer (5'-GTAAAACGACGGCCAGT-3') and the M13 reverse primer (5'-CAGGAAACAGCTATGAC-3'), which hybridize to 
pGEM-T Easy DNA. A consensus sequence was created using the software BioEdit Sequence Alignment Editor (version 7.0.5.3). 16S rRNA gene sequences were analysed using BLAST (National Center for Biotechnology Information; http://www.ncbi.nml.nih.gov) by running the BLASTN program (Altschul et al., 1997).

Identification of degradation intermediates via GC/MS analyses. The compositions of the cell-free supernatants of cultures and of the standards of important OSCs were determined upon methylation after lyophilization in the presence of $15 \%(\mathrm{v} / \mathrm{v})$ sulphuric acid $\left(\mathrm{H}_{2} \mathrm{SO}_{4}\right)$ by gas chromatographic analysis of the resulting methylesters, as described previously (Brandl et al., 1988; Timm et al., 1990).

Transposon mutagenesis of $\boldsymbol{R}$. erythropolis MI2. For transposon mutagenesis, the suicide vectors pFAJ2571 and pFAJ2527 (Nagy et al., 1997) were transferred into the chloramphenicol-susceptible isolate $R$. erythropolis MI2 by electroporation. Transposon-induced mutants were selected on MSM agar plates containing $50 \mu \mathrm{g}$ chlorampheni$\mathrm{col} \mathrm{ml}{ }^{-1}\left(\mathrm{MSM}^{\mathrm{Cm}}\right)$ and $0.2 \%(\mathrm{w} / \mathrm{v})$ sodium gluconate (master plates). Putative mutants were transferred in a coordinated pattern to $\mathrm{MSM}^{\mathrm{Cm}}$ agar plates containing $0.2 \%(\mathrm{w} / \mathrm{v})$ DTDB (selection plates) and corresponding master plates for further analysis.

\section{RESULTS}

\section{Utilization of DTDB, DTDP or TDP by available bacteria}

To examine different bacterial strains (Table 1) for their ability to utilize DTDB, DTDP or TDP as the sole source of carbon and energy, growth experiments were conducted with solid MSM containing $0.2 \%(\mathrm{w} / \mathrm{v})$ of the respective OSCs. However, none of the strains listed in Table 1 was able to grow on any of the three OSCs as the sole carbon and energy source.

\section{Isolation and characterization of bacterial strains utilizing DTDB, DTDP or TDP as sole source of carbon and energy}

Isolation and characterization of some DTDP- and TDPutilizing bacteria have been previously reported (Bruland et al. 2009a, b; Toups et al., 2009; Wübbeler et al. 2006); the same enrichment procedure was employed during this study to isolate novel bacterial strains capable of using DTDB or DTDP as the sole source of carbon and energy. Enrichment cultures were done under mesophilic and aerobic conditions in MSM (Schlegel et al., 1961b), and were inoculated with diverse samples from polluted or sulphur-containing soils and which contained one of the OSCs mentioned above. These enrichment cultures yielded many bacterial isolates exhibiting morphologically different colonies on OSC-containing MSM agar plates. The evaluation of API test strips and of $16 \mathrm{~S}$ rRNA gene sequences of initially interesting strains revealed high similarities of the isolates to strains of species belonging to the genera Alcaligenes, Bordetella, Cupriavidus, Klebsiella and Rhodococcus (Table 2). We focused on the most promising isolates regarding growth rate, susceptibility to antibiotics (Table 2) and predicted non-pathogenicity to humans. Isolates affiliated into genera with human pathogen species, such as Alcaligenes, Bordetella and Klebsiella, were not considered further. The other isolates were later characterized in more detail and are presented in Table 2. Isolates from very recent studies also comprising members of new taxa, such as A. mimigardefordensis strain DPN7 ${ }^{\mathrm{T}}$ (Gibello et al., 2009; Wübbeler et al., 2006) and Pseudorhodoferax soli strain TBEA3 (Bruland et al., 2009b), were also investigated (Table 2).

All isolates listed in Table 2 used acetate, propionate and gluconate as the sole carbon and energy source for growth. They all, except strain BEA3, also grew with butyrate. Fructose was not utilized by strain DPN7 ${ }^{\mathrm{T}}$; strains SFWT, TBEA $3^{\mathrm{T}}$, TBEA6 and DBR3 showed no growth on glucose. None of the strains listed in Table 2 grew with dibutylsulphide, ethanol, 2-mercaptopropionate, 3MP, 4mercapto-1-butanol or $\gamma$-thiobutyrolactone (TBL) as the sole carbon and energy source for growth.

Interestingly, none of these OSC-utilizing isolates was able to use either of the two other OSCs investigated in this study as a carbon source for growth. For example, no DTDB-utilizing isolate was able to grow on the close structural analogue DTDP (Fig. 1c) or on TDP (Fig. 1a).

\section{Isolates able to utilize TDP or DTDP as the sole carbon and energy source}

$V$. paradoxus strain TBEA6, V. paradoxus strain SFWT and the recently classified P. soli (Bruland et al., 2009a, b) are, besides Schlegelella thermodepolymerans (Elbanna et al., 2003), the only known characterized strains using TDP as the sole source of carbon and energy (Table 2).

The degradation and utilization of DTDP are on the one hand accomplished by four closely related Gram-positive bacteria, which were all affiliated to the species $R$. erythropolis (strains BEA1, BEA2, BEA3 and BEA4). On the other hand, five Gram-negative strains with the capability to grow with DTDP as a carbon source have been characterized: two strains of the genus Pseudomonas, one Bordetella species (Toups et al., 2009), and two further Gram-negative bacteria affiliated as Cupriavidus taiwanensis strain BEA5c or A. mimigardefordensis strain $\mathrm{DPN}^{\mathrm{T}}$ (Wübbeler et al., 2006) (Table 2).

\section{Biodegradation of DTDB}

This study reports for the first time, to our knowledge, the microbial utilization of DTDB. Apparently, DTDB is used as the sole source of carbon and energy exclusively by some novel isolated strains of R. erythropolis (Table 2). No representatives of other genera were obtained under the enrichment conditions employed in this study. Growth of these Gram-positive natural isolates was initially observed on solidified MSM agar plates and was then confirmed in 

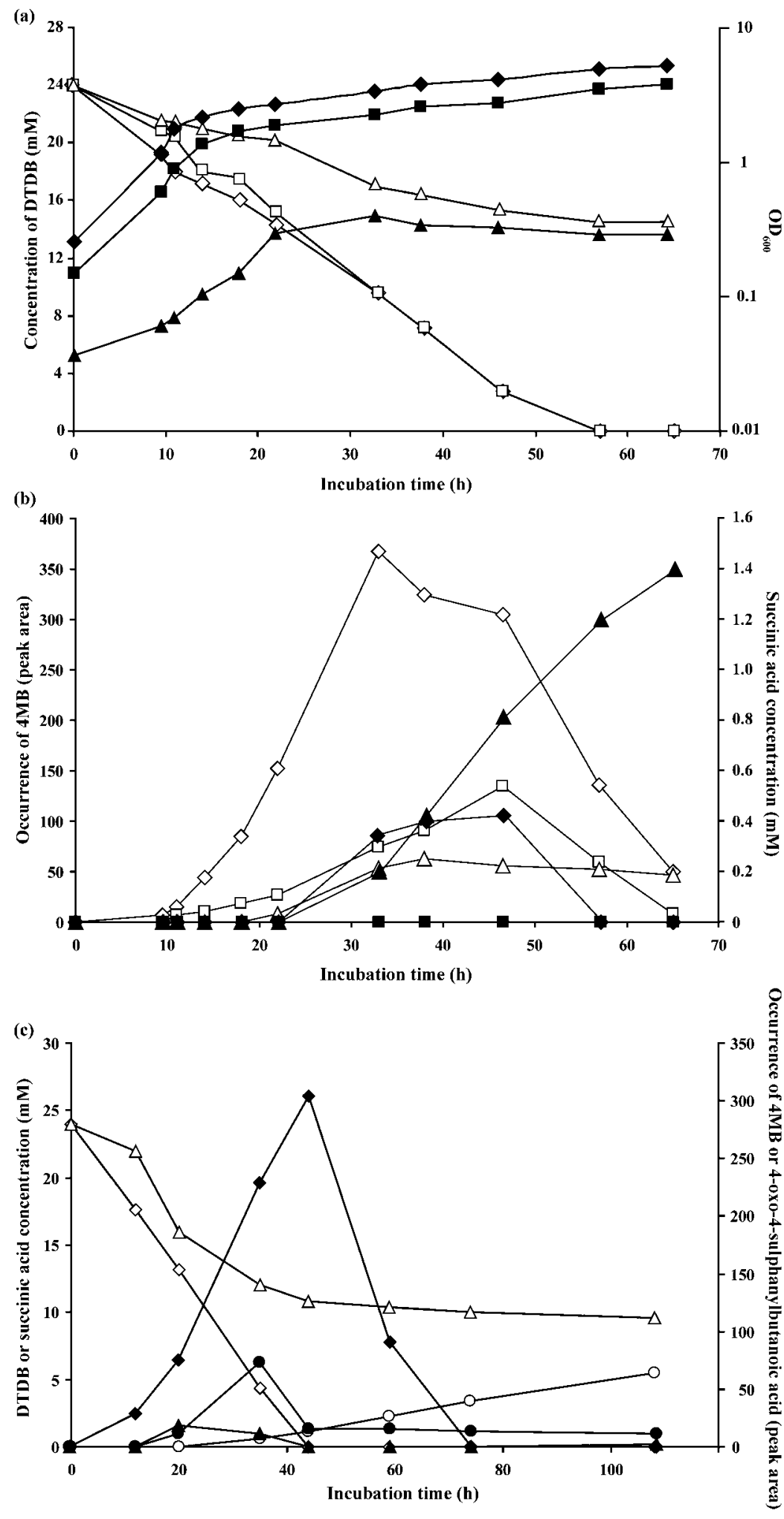

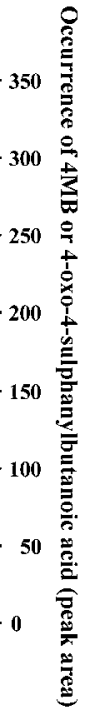

liquid MSM, where an increase in turbidity was accompanied by a concomitant decrease in DTDB, as shown with $R$. erythropolis strain MI2 as an example in Fig. 2(a).
Controls (cultures without inoculum or without a carbon source) did not show any decrease in the DTDB concentration or increase of the optical density, respectively 
Fig. 2. Degradation of DTDB by $R$. erythropolis strain MI2 and by the DTDB-negative mutant 1.7/17. Cells were cultivated in 1 I flasks without baffles on a rotary shaker at $30{ }^{\circ} \mathrm{C}$ and 120 r.p.m. in MSM containing either $24 \mathrm{mM}$ DTDB as the sole carbon source or $24 \mathrm{mM}$ DTDB in addition to $0.5 \%(\mathrm{w} / \mathrm{v})$ sodium gluconate. Analyses of the cell-free supernatants were done by GC and GC/MS. Because 4-oxo-4-sulphanylbutanoic acid and 4MB are not commercially available, they could not be applied as controls, standards or in calibrations. Therefore, detection of these compounds is shown as 'peak area', depending on the respective signal intensity. (a) Utilization of DTDB and increase of turbidity. Symbols: $\diamond$, consumption of DTDB as the sole carbon source by $R$. erythropolis strain MI2; $\square$, consumption of DTDB by $R$. erythropolis strain MI2 during cultivation with sodium gluconate as a second carbon source; $\triangle$, consumption of DTDB by mutant 1.7/17 during cultivation with sodium gluconate as a second carbon source; $>$, increase of turbidity in cultures of $R$. erythropolis strain MI2 during growth with DTDB as the sole carbon source; $\mathbf{\square}$, increase of turbidity in cultures of $R$. erythropolis strain MI2 during growth with DTDB and sodium gluconate as carbon sources; $\boldsymbol{\Lambda}$, increase of turbidity in cultures of mutant 1.7/17 during growth with DTDB and sodium gluconate as carbon sources. (b) Detection of the degradation intermediates $4 \mathrm{MB}$ and succinic acid. Symbols: $\diamond$, concentration of $4 \mathrm{MB}$ during degradation of DTDB as the sole carbon source by cells of $R$. erythropolis strain $\mathrm{MI}$; $\square$, concentration of $4 \mathrm{MB}$ during degradation of DTDB in addition to sodium gluconate as a second carbon source by cells of $R$. erythropolis strain MI2; $\triangle$, concentration of $4 \mathrm{MB}$ during degradation of DTDB in addition to sodium gluconate as a second carbon source by cells of mutant $1.7 / 17 ;>$, concentration of succinic acid during degradation of DTDB as sole carbon source by cells of $R$. erythropolis strain MI2; $\mathbf{n}$, concentration of succinic acid during degradation of DTDB and sodium gluconate as carbon sources by cells of $R$. erythropolis strain $\mathrm{MI} 2 ; \boldsymbol{\Lambda}$, concentration of succinic acid during degradation of DTDB and sodium gluconate as carbon sources by cells of mutant 1.7/17. (c) Subsequent analyses of cell-free supernatants of $R$. erythropolis strain MI2 and mutant 1.7/17 in MSM with $24 \mathrm{mM} \mathrm{DTDB}$ as the sole carbon source after preincubation for $48 \mathrm{~h}$ in MSM with $0.5 \%(\mathrm{w} / \mathrm{v})$ sodium gluconate. Symbols: $\diamond$, consumption of DTDB by $R$. erythropolis strain MI2; $\triangle$, consumption of DTDB by mutant $1.7 / 17 ; 4 \mathrm{MB}, R$. erythropolis strain MI2; $\mathbf{\Delta}$, concentration of $4 \mathrm{MB}$, mutant $1.7 / 17 ; \bigcirc$, concentration of succinic acid, mutant $1.7 / 17 ;$, concentration of 4 -oxo-4-sulphanylbutanoic acid, mutant $1.7 / 17$.

(data not shown). The following experiments were accomplished to unravel the catabolism of DTDB in $R$. erythropolis strain MI2.

\section{Isolation and phenotypic characterization of transposon-induced mutants of $R$. erythropolis MI2 impaired in DTDB degradation}

All further studies were done with $R$. erythropolis strain MI2, because of the strain's stable susceptibility to chloramphenicol. Therefore, this isolate was subjected to transposon mutagenesis employing the suicide vector plasmids pFAJ2571 and pFAJ2572 (Nagy et al., 1997), conferring chloramphenicol resistance to the recipient. This mutagenesis aimed at the generation of mutants with defective growth on DTDB to investigate the DTDB catabolism. Insertions of Tn5561X1 and Tn5561X2 into the genomes of these mutants were confirmed by PCR using primers hybridizing to ist $B$ (forward primer) and $c m r$ (reverse primer). Genomic DNA of the wild-type was used as negative control.

Among 7500 analysed mutants, three were identified with a defect in the utilization of DTDB. Two mutants (designated 1.7/17 and 2.3/36) exhibited fully impaired growth on MSM agar plates containing DTDB as the sole carbon source, and were referred to as DTDB-negative mutants. One mutant (designated 1.5/45) exhibited slower growth on the same plates,

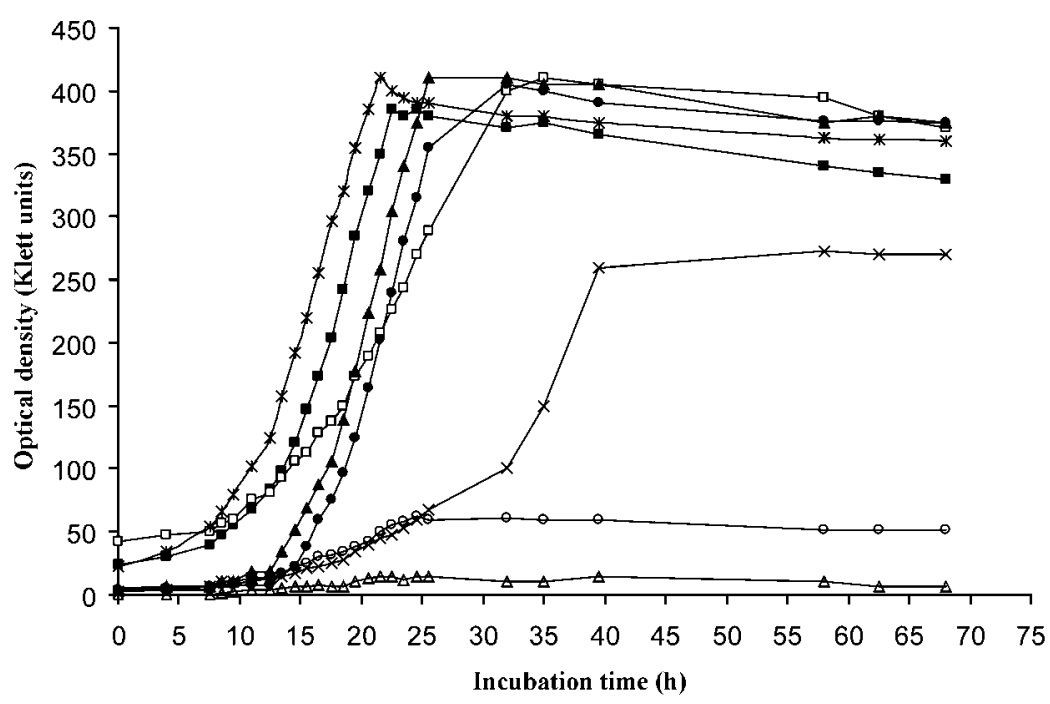

Fig. 3. Utilization of DTDB and sodium gluconate by the wild-type and transposoninduced mutants of $R$. erythropolis strain $\mathrm{MI} 2$ affected in growth on DTDB. Cells were cultivated in $50 \mathrm{ml}$ Klett flasks with baffles on a rotary shaker at $30{ }^{\circ} \mathrm{C}$ and 120 r.p.m. in liquid MSM containing $0.2 \%(\mathrm{w} / \mathrm{v})$ DTDB or $0.2 \%(\mathrm{w} / \mathrm{v})$ sodium gluconate, respectively, as the sole carbon sources. Symbols: $\mathbf{\square}$, wildtype, gluconate; $\square$, wild-type, DTDB; $\mathbf{0}$, mutant 2.3/36, gluconate; $\bigcirc$, mutant 2.3/36, DTDB; $\boldsymbol{\Delta}$, mutant $1.7 / 17$, gluconate; $\triangle$, mutant 1.7/17, DTDB; *, mutant 1.5/45, gluconate; $\times$, mutant $1.5 / 45$, DTDB. 
and was therefore referred to as a DTDB-leaky mutant. All three mutants grew otherwise like the wild-type. The growth behaviour of these mutants and the wild-type on liquid MSM with sodium gluconate and DTDB is compared in Fig. 3.

\section{Identification of DTDB degradation metabolites in cultures of the wild-type and of the three DTDB- defective mutants of $R$. erythropolis strain MI2}

GC/MS analysis of the lyophilized cell-free supernatant obtained from wild-type cultures of $R$. erythropolis strain MI2 identified, in addition to DTDB, the predicted symmetrical cleavage compound $4 \mathrm{MB}$ and succinic acid as discharged degradation intermediates of DTDB catabolism (Fig. 2a, b). Furthermore, no increase of sulphite or sulphate was detected in any of the cultures; instead, sulphur in excess of its need as a sulphur source was released as volatile hydrogen sulphide, detected by the occurrence of a black precipitate on the test strips due to the conversion of lead(II) acetate into lead(II) sulphide. In contrast, no hydrogen sulphide could be detected in the media of DTDB cultures of $R$. erythropolis strain MI2 at any time; therefore, the concentration of solubilized hydrogen sulphide in the medium was always below $3 \mu \mathrm{M}$ (the lower limit of detection). Volatile or solubilized hydrogen sulphide could not be detected in cultures of this bacterium utilizing gluconate as the sole carbon source.

To identify intermediates that accumulate during catabolism of DTDB, the three mutants and the wild-type of $R$. erythropolis strain MI2 were cultivated in MSM containing $0.5 \%(\mathrm{w} / \mathrm{v})$ sodium gluconate as utilizable carbon source; $24 \mathrm{mM}$ DTDB was added to each culture after $24 \mathrm{~h}$ of incubation. Samples were withdrawn every day, and the cell-free supernatant obtained from each culture was analysed by GC/MS. Within 4 days of cultivation, the wild-type and the DTDB-leaky mutant $1.5 / 45$ consumed all available DTDB (data not shown). The easily detectable $4 \mathrm{MB}$, which is most likely the product of symmetrical cleavage of DTDB, was identified as an abundant intermediate in the supernatants of the wild-type (Fig. 4b), of the DTDB-negative mutant 1.7/17 (Fig. 4a) and of the DTDB-leaky mutant 1.5/45 (data not shown). Moreover, succinic acid (Fig. 4) and, in very low

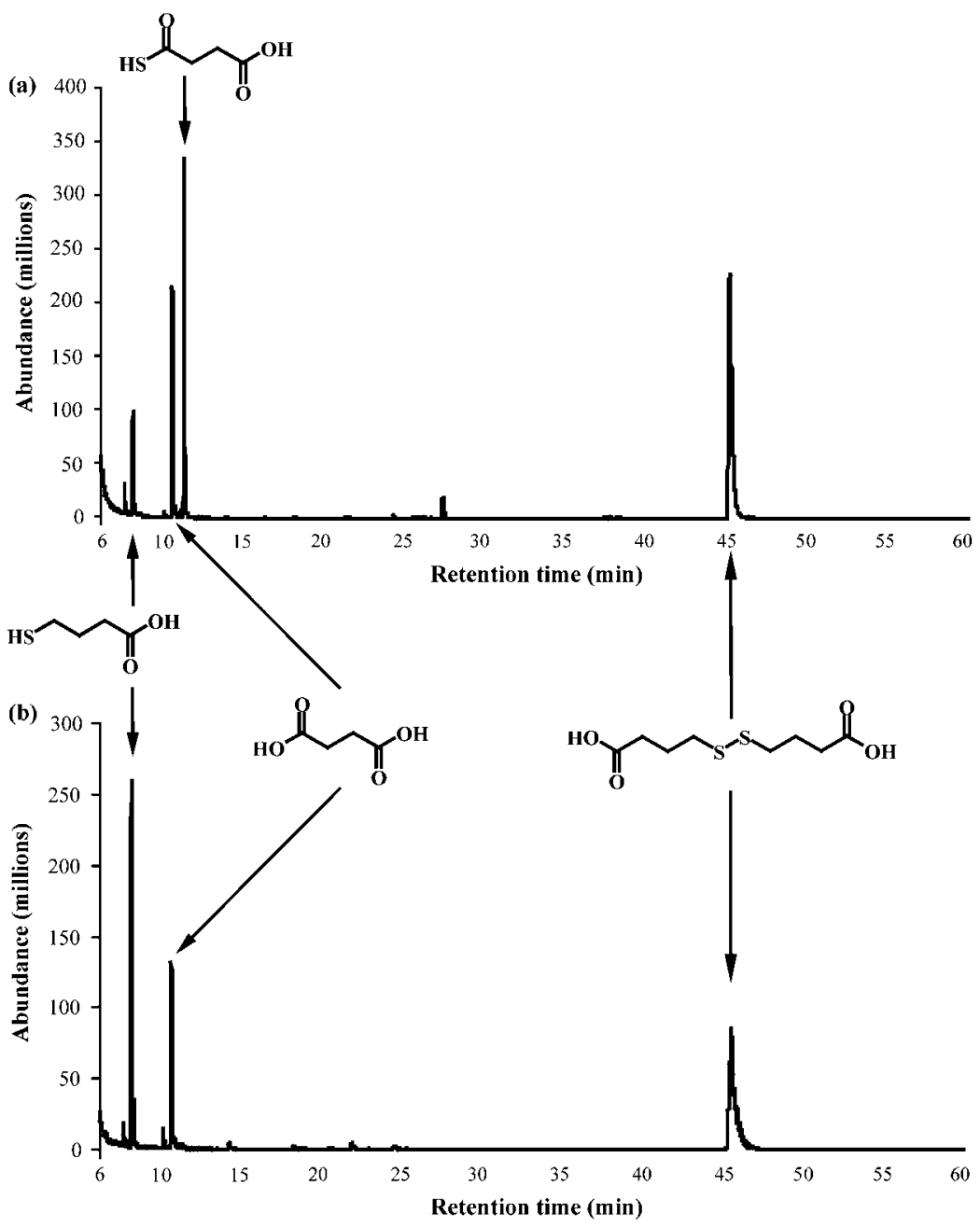

Fig. 4. GC/MS analyses of DTDB-degradation intermediates. Chromatograms of the wildtype and of mutant $1.7 / 17$ of $R$. erythropolis strain MI2 are compared. Cells were cultivated in $250 \mathrm{ml}$ flasks without baffles on a rotary shaker at $30{ }^{\circ} \mathrm{C}$ and 120 r.p.m. in MSM containing $0.5 \%(\mathrm{w} / \mathrm{v})$ sodium gluconate; after $24 \mathrm{~h}$ of cultivation, $24 \mathrm{mM}$ DTDB was added. (a) Cell-free supernatant of mutant 1.7/17, and (b) cell-free supernatant of the wild-type; both were incubated for $48 \mathrm{~h}$ after inoculation. Besides DTDB, the intermediates $4 \mathrm{MB}$, 4oxo-4-sulphanylbutanoic acid and succinic acid were separated in the respective chromatograms and identified. 
concentrations, also fumaric acid (not highlighted in Fig. 4) were detected. The analysis of the DTDB-negative mutant 2.3/36 revealed neither a detectable decrease in the DTDB concentration in the supernatant nor any degradation intermediates (data not shown). In contrast, the DTDB-negative mutant 1.7/17 depleted the DTDB concentration down to $2 \mathrm{mM}$ within 7 days of incubation, whereas succinic acid was accumulated and could be detected at a high concentration (up to $21.6 \mathrm{mM}$ ) in the medium. Furthermore, considerable amounts of an unexpected OSC could be observed in the culture supernatant; it was identified as 4-oxo-4-sulphanylbutanoic acid by GC/MS analyses (Fig. 4a).

Cell pellet analysis of all four strains revealed barely detectable amounts of $4 \mathrm{MB}$ as the only identifiable intermediate.

\section{Comparison of $\boldsymbol{R}$. erythropolis strain MI2 and mutant 1.7/17 with respect to growth behaviour and degradation intermediates}

Well-established precultures incubated in MSM containing $0.5 \%(\mathrm{w} / \mathrm{v})$ sodium gluconate were used to inoculate the main cultures of both strains. Growth behaviour in MSM containing $24 \mathrm{mM}$ DTDB and $0.5 \%(\mathrm{w} / \mathrm{v})$ sodium gluconate was compared also with that of the wild-type cultivated in MSM containing $24 \mathrm{mM}$ DTDB as the sole carbon source, and is presented in Fig. 2(a). In less than $60 \mathrm{~h}$, both wild-type cultures degraded all available DTDB, thereby generating and discharging significant amounts of $4 \mathrm{MB}$ (Fig. $2 \mathrm{~b})$. In the supernatant of the wild-type culture, containing sodium gluconate as a second carbon source, no succinic acid was detectable (Fig. 2b). Despite utilization of $7.4 \mathrm{mM}$ DTDB, growth of mutant $1.7 / 17$ was weak; the turbidity increased up to an $\mathrm{OD}_{600}$ of only 0.4 (Fig. 2a), presumably due to the inhibitory effects of the accumulation of DTDBdegradation intermediates. However, $4 \mathrm{MB}$ was detectable and the accumulation of succinic acid in the supernatant could be verified (Fig. 2b). Surprisingly, no 4-oxo-4sulphanylbutanoic acid was detectable under these cultivation conditions, although solubilized hydrogen sulphide at a concentration of up to $12 \mu \mathrm{M}$ (after $18 \mathrm{~h}$ of incubation) was detected in the culture supernatant. Volatile hydrogen sulphide was always produced by $R$. erythropolis strain MI2 and mutants if DTDB was used as a carbon source.

An additional modified cultivation variant was applied to the wild-type and mutant $1.7 / 17$. Cells of both strains were preincubated in MSM with $0.5 \%(\mathrm{w} / \mathrm{v})$ sodium gluconate for $48 \mathrm{~h}$ and then transferred into fresh media containing $24 \mathrm{mM}$ DTDB as the sole carbon source. The wild-type utilized $24 \mathrm{mM}$ DTDB within approximately $40 \mathrm{~h}$ (Fig. 2c). $4 \mathrm{MB}$ was detected during degradation, but neither succinic acid nor hydrogen sulphide was detectable in the supernatant. Mutant 1.7/17 degraded $14.4 \mathrm{mM}$ DTDB within $108 \mathrm{~h}$, and produced easily detectable amounts of $4 \mathrm{MB}$ and 4-oxo-4-sulphanylbutanoic acid in the supernatant during this period (Fig. 2c). Accumulation of hydrogen sulphide in the supernatant was detected only during the first $24 \mathrm{~h}$ of incubation, with a maximum of $18 \mu \mathrm{M}$ after $20 \mathrm{~h}$. Subsequently, the concentration of succinic acid increased steadily (Fig. 2c).

\section{Cleavage of DTDP by $R$. erythropolis strain MI2 and conversion of DTDB by $A$. mimigardefordensis strain DPN7 ${ }^{\top}$}

The isolates listed in Table 2 were tested for their ability to convert a particular 'non-utilizable' OSC, DTDB, DTDP or TDP, if cultivated in the presence of a second easily degradable carbon source, e.g. sodium gluconate. No strain yielded any evidence for cleavage or other conversions of the added foreign OSC, as verified by GC and GC/MS. On the other hand, the respective 'non-utilizable' OSC did not exert any toxic effect on the cells if applied at concentrations lower than $1 \%(\mathrm{w} / \mathrm{v})$.

To elucidate whether the substrate specificity of strains MI2 and DPN7 results from inducing and regulatory effects, the experiments were done in a modified way. In these experiments, the 'non-utilizable' OSC was added to cultures containing the 'utilizable' OSC. Therefore, both strains were cultivated in MSM containing $20 \mathrm{mM}$ DTDP plus $20 \mathrm{mM}$ DTDB. Samples were withdrawn every $48 \mathrm{~h}$ and analysed for the occurrence of putative cleavage products by GC/MS (Fig. 5). After 4 days of incubation, up to $4.5 \mathrm{mM} 3 \mathrm{MP}$ was detected as the cleavage product of DTDP when cells of strain MI2 were grown with DTDP plus DTDB. In contrast, when cells of strain DPN7 were cultivated for 6 days in MSM containing DTDB plus DTDP, TBL, which is the lactone of $4 \mathrm{MB}$, was detected at a concentration of up to $2.6 \mathrm{mM}$. Interestingly, A. mimigardefordensis strain $\mathrm{DPN}^{\mathrm{T}}$ did not release $4 \mathrm{MB}$ into the medium.

\section{DISCUSSION}

Although a variety of bacterial strains (Table 1) with uncommon degradation capabilities was cultivated on minimal media containing one of the OSCs, no growth of any of these strains was detected. In fact, only a few cultivable strains are able to utilize DTDB, DTDP or TDP as the sole sources of carbon and energy (Table 2). Furthermore, all DTDB-degrading strains could not utilize DTDP or TDP, while none of the DTDP-utilizing strains grew with DTDB or TDP, and no TDP-catabolizing strain utilized DTDB or DTDP. The inability to grow on the respective non-utilizable OSC is probably caused by an inability to catalyse the initial cleavage steps or to transport the compounds into the cells, or the absence of other means of conversion, if the cells are not cultivated in the presence of a utilizable OSC. Consequently, $R$. erythropolis strain MI2 and A. mimigardefordensis strain $\mathrm{DPN}^{\mathrm{T}}$ can cleave the respective non-utilizable disulphide, when DTDB (strain MI2) or DTDP (strain DPN7 ${ }^{\mathrm{T}}$ ) are at the same time available for growth. This indicates that the 


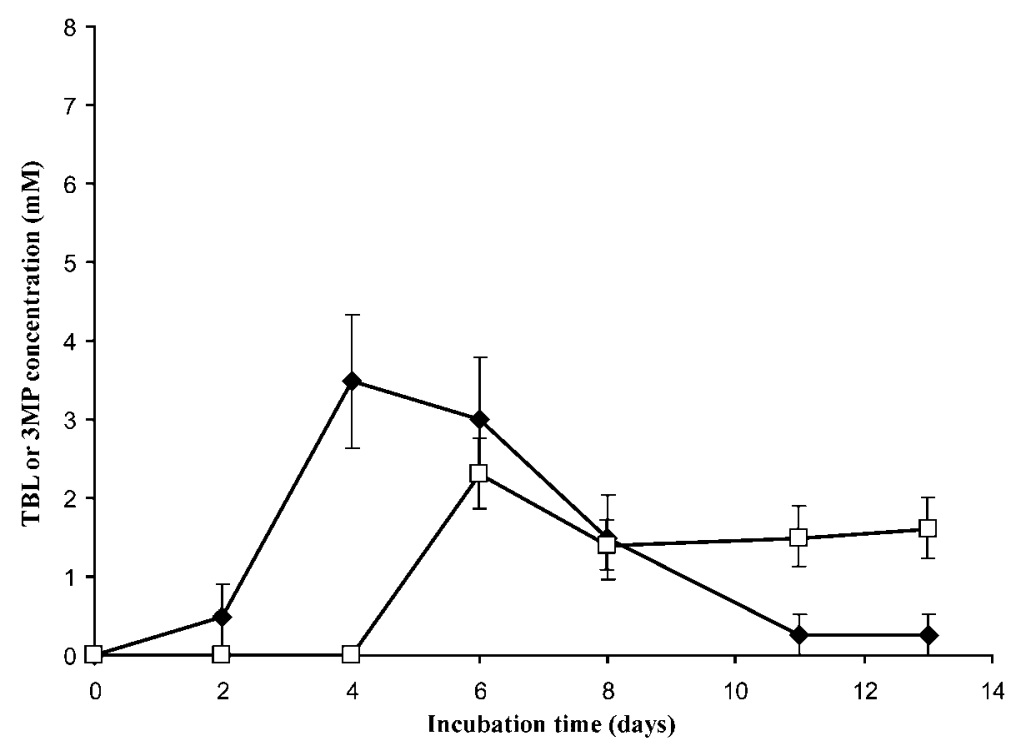

Fig. 5. Conversion of DTDP by $R$. erythropolis strain MI2 and of DTDB by A. mimigardefordensis strain $\mathrm{DPN7}^{\top}$, and occurrence of the respective cleavage products $3 \mathrm{MP}$ and TBL. Cells were cultivated in $250 \mathrm{ml}$ flasks without baffles on a rotary shaker at $30{ }^{\circ} \mathrm{C}$ and 120 r.p.m. in MSM containing $20 \mathrm{mM}$ DTDB and $20 \mathrm{mM}$ DTDP. Analyses of the OSCs in the cell-free supernatants were done by GC and GC/MS. Standard deviations based on three measurements are indicated. $\square$, TBL; , 3MP.

enzymes catalysing the first steps in the catabolism of DTDB and DTDP also react with the other substrate in $R$. erythropolis strain MI2 and A. mimigardefordensis strain $\mathrm{DPN7}^{\mathrm{T}}$, respectively.

A. mimigardefordensis strain $\mathrm{DPN}^{\mathrm{T}}$ presumably converts $4 \mathrm{MB}$ to TBL due to the high toxicity of $4 \mathrm{MB}$ to living cells (Held \& Biaglow, 1994). 4MB could be converted to the corresponding lactone TBL, assuming either the involvement of a lipase (Gupta et al., 2004) or a reaction analogous to the error-editing process catalysed by certain tRNA synthetases, converting the non-protein amino acid homocysteine (which is a close structural analogue of $4 \mathrm{MB})$ to the thioester homocysteine-thiolactone (Jakubowski \& Fersht, 1981; Jakubowski \& Goldman, 1992).

It became evident in this study that all three OSCs are biodegradable and are used as the sole carbon and energy sources for growth by a few soil micro-organisms with exclusive degradation abilities. Whether the utilization depends on rare functions and low substrate specificities of well-known enzymes, or whether the degrading enzymes are specifically synthesized for the degradation of one particular OSC has to be further investigated. Whereas different bacterial species could be isolated using TDP or DTDP as the sole carbon source, the degradation of DTDB seems to be restricted to strains of $R$. erythropolis alone. Rhodococci are widespread in natural habitats, and the members of this genus are known for their broad catabolic diversity and unique catabolic capabilities (Bell et al., 1998). R. erythropolis cells contain a large set of enzymes, which allow the cells to perform enzyme-catalysed reactions such as oxidations, dehydrogenations, epoxidations, hydrolysis, hydroxylations and dehalogenations (de Carvalho et al., 2005). Members of the genus Rhodococcus also have unique abilities to cleave carbon-sulphur bonds without affecting the carbon-carbon bond. R. erythropolis
IGTS8 is therefore used as model organism to study the microbial desulphurization of fossil fuels via a biological system (Grossman et al., 2001). Furthermore, the industrial production of acrylamide is an important biotechnological application of rhodococci (Yamada \& Kobayahashi, 1996).

Novel pathways for the degradation of DTDP and TDP have been recently presented (Bruland et al., 2009a; Wübbeler et al., 2008). Based on this knowledge and on the experimental data on the phenotypic and physiological characteristics of DTDB-defective mutants of $R$. erythropolis strain MI2 revealed in this study, we propose the putative degradation pathway of DTDB shown in Fig. 6. DTDB degradation is most likely initiated by a symmetrical cleavage performed by putative disulphide oxidoreductases, thereby yielding two molecules of $4 \mathrm{MB}$. This compound is more toxic than the other mercaptoalkanoates used in this study, as documented in the past during synthesis of PTEs (Lütke-Eversloh \& Steinbüchel, 2003), and as described for free thiols in many biochemical processes (Held \& Biaglow, 1994). 4MB is proposed as an intermediate, since it is easily detectable in supernatants of cultures with DTDB as the carbon source (Figs 2 and 4). For this initial step, the participation of an oxidoreductase is likely. Flavoprotein disulphide reductases catalyse, for example, the reduction of compounds which are structurally related and linked by disulphide bonds (Williams, 1992). In the catabolism of DTDP, the reduction of the disulphide bond is most likely catalysed by a dihydrolipoamide dehydrogenase homologous enzyme with enhanced substrate specificity (Wübbeler et al., 2008; J. H. Wübbeler and others, unpublished results). Considering the available data on the completely sequenced genome of Rhodococcus strain RHAl (McLeod et al., 2006), more than 500 ORFs that encode reductases, including six genes encoding paralogues of dihydrolipoamide dehydrogenases, are present. Moreover, A. mimigardefordensis strain DPN7 and R. erythropolis strain MI2 were both able to cleave 


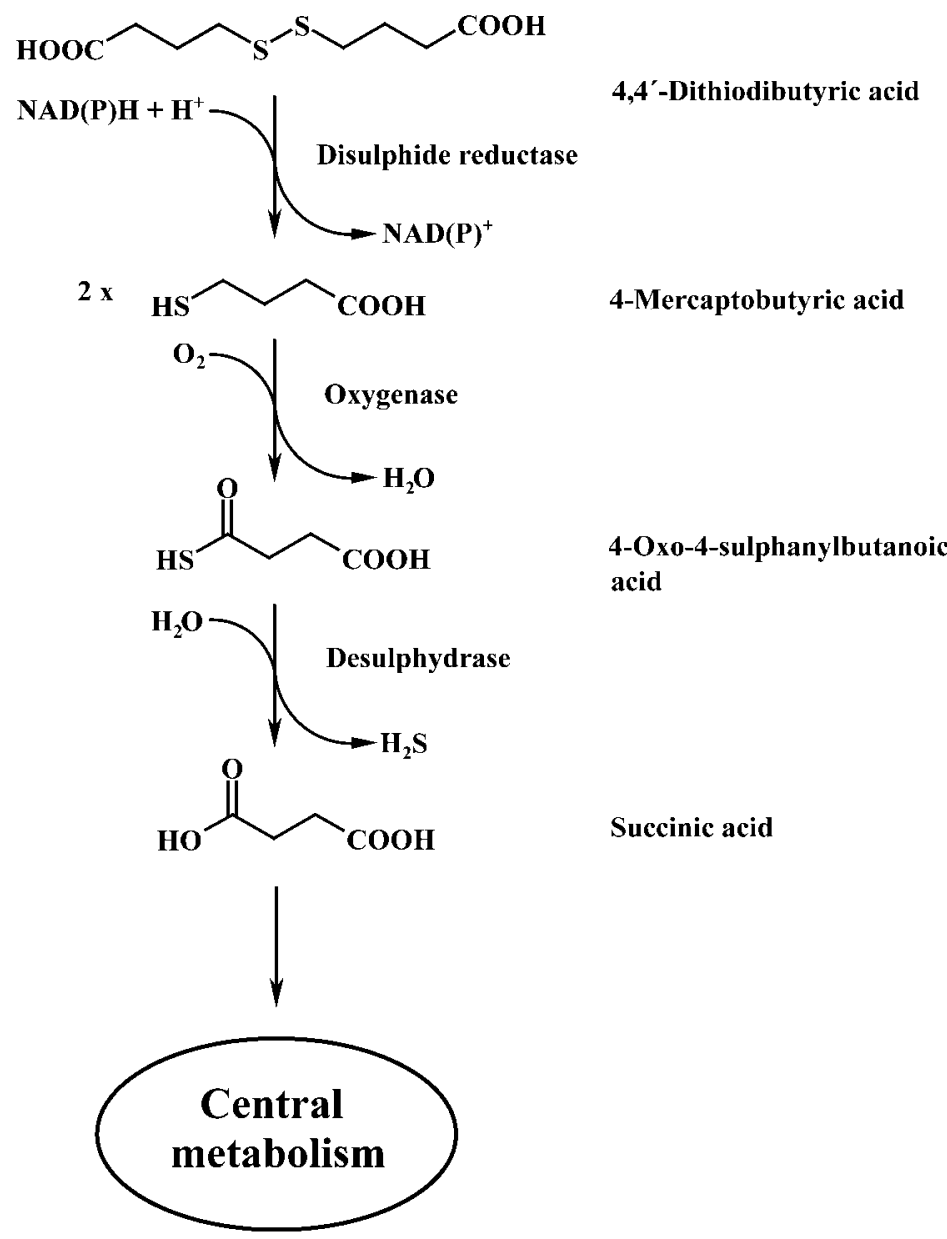

Fig. 6. Putative pathway for the degradation of DTDB in $R$. erythropolis strain MI2. Initially, DTDB is putatively cleaved into two molecules of $4 \mathrm{MB}$, which is then probably oxidized by an oxygenase, thereby yielding 4-oxo-4-sulphanylbutanoic acid. Afterwards, the sulphur moieties could be removed as volatile hydrogen sulphide by a putative desulphydrase. The resulting succinic acid is then further metabolized.

the disulphides (DTDB and DTDP) symmetrically, if incubated under inducing conditions, as shown in Fig. 5.

The second proposed step in the catabolism of DTDB is the oxidation of $4 \mathrm{MB}$ to the corresponding carboxylic acid 4-oxo-4-sulphanylbutanoic acid. This compound was detected in the culture supernatants of the DTDB-negative mutant $1.7 / 17$ (Figs $2 \mathrm{c}$ and $4 \mathrm{a}$ ). The reaction is presumably performed by an oxygenase, and the subsequent step is thought to be the abstraction of the sulphur, catalysed by a putative desulphydrase. Approximately 200 ORFs encoding oxygenases and two ORFs encoding desulphydrases have been identified in Rhodococcus strain RHA1 (McLeod et al., 2006). The putative desulphydrase of $R$. erythropolis strain MI2 presumably converts 4-oxo-4-sulphanylbutanoic acid into volatile hydrogen sulphide and succinic acid; the latter can be further metabolized in the central metabolism via the citric acid cycle. All previously proposed products have been proven to be intermediates.

In conclusion, microbial utilization apparently converts DTDB into two molecules of $4 \mathrm{MB}$, which are then further metabolized. After identification and characterization of the genes involved, the construction of metabolically engineered strains suitable for production of $4 \mathrm{MB}$-containing PTEs should become possible. The system of transpo- son mutagenesis (Nagy et al., 1997) used in this study, which was the only available and applicable method at the time, is not suitable for the genotypic characterization of transposon-induced mutants, as also described elsewhere (Sallam et al., 2006). However, a promising system for the construction of transposon mutant libraries of strains of $R$. erythropolis has been developed just recently (Sallam et al., 2007). This tool should be employed for the complete elucidation of DTDB catabolism. Subsequently, the successful heterologous expression of genes of the butyrate kinase/phosphotransbutyrylase/PHA synthase (BPEC) pathway (Liu \& Steinbüchel, 2000) in genetically manipulated $R$. erythropolis could yield PTE with $4 \mathrm{MB}$ building blocks. As already mentioned, detailed knowledge of the DTDB- or $4 \mathrm{MB}$-degrading pathways is a prerequisite to engineer the optimized production strains. The data presented and the anticipated findings are therefore especially supportive of the prospective synthesis of such interesting polymers.

\section{ACKNOWLEDGEMENTS}

The authors thank Matthias Arenskötter for helpful discussions and instructions in methods. 


\section{REFERENCES}

Altschul, S. F., Madden, T. L., Schäffer, A. A., Zhang, J., Zhang, Z., Miller, W. \& Lipman, D. J. (1997). Gapped BLAST and PSI-BLAST: a new generation of protein database search programs. Nucleic Acids Res 25, 3389-3402.

Appel, R. B., Tomlinson, I. A. \& Hill, I. (1995). New reagents for the reductive quenching of ozonolysis reactions. Synth Commun 25, 3589-3595.

Bell, K. S., Philp, J. C., Aw, D. W. J. \& Christofi, N. (1998). The genus Rhodococcus. J Appl Microbiol 85, 195-210.

Birnboim, H. C. \& Doly, J. (1979). A rapid alkaline extraction procedure for screening recombinant plasmid DNA. Nucleic Acids Res 7, 1513-1523.

Brandl, H., Gross, A., Lenz, R. W. \& Fuller, R. C. (1988). Pseudomonas oleovorans as a source of poly( $\beta$-hydroxyalkanoates) for potential applications as biodegradable polyesters. Appl Environ Microbiol 54, 1977-1982.

Bruland, N., Wübbeler, J. H. \& Steinbüchel, A. (2009a). 3Mercaptopropionate dioxygenase, a cysteine dioxygenase homologue, catalyzes the initial step of 3-mercaptopropionate catabolism in the 3,3' -thiodipropionic acid degrading bacterium Variovorax paradoxus. J Biol Chem 284, 660-672.

Bruland, N., Bathe, S., Willems, A. \& Steinbüchel, A. (2009b). Pseudorhodoferax soli gen. nov., sp. nov., and Pseudorhodoferax caeni sp. nov., two members of the class Betaproteobacteria belonging to the family Comamonadaceae. Int J Syst Evol Microbiol 59, 2702-2707.

Codognoto, L., Winter, E., Paschoal, J. A. R., Suffredini, H. B., Cabral, M. F., Machado, S. A. S. \& Rath, S. (2007). Electrochemical behavior of dopamine at a 3,3'-dithiodipropionic acid self-assembled monolayers. Talanta 72, 427-433.

de Carvalho, C. C. C. R. \& da Fonseca, M. M. R. (2005). The remarkable Rhodococcus erythropolis. Appl Microbiol Biotechnol 67, 715-726.

Desomer, J., Dhaese, P. \& van Montagu, M. (1990). Transformation of Rhodococcus fascians by high-voltage electroporation and development of $R$. fascians cloning vectors. Appl Environ Microbiol 56, 2818-2825.

Elbanna, K., Lütke-Eversloh, T., Van Trappen, S., Mergaert, J., Swings, J. \& Steinbüchel, A. (2003). Schlegelella thermodepolymerans gen. nov., sp. nov., a novel thermophilic bacterium that degrades poly(3-hydroxybutyrate-co-3-mercaptopropionate). Int J Syst Evol Microbiol 53, 1165-1168.

Gerhardt, P., Murry, R. G. E., Wood, W. A. \& Krieg, N. R. (1994). Methods for General and Molecular Bacteriology. Washington, DC: American Society for Microbiology.

Gibello, A., Vela, A. I., Martin, M., Barra-Caracciolo, A., Grenni, P. \& Fernández-Garayzábal, J. F. (2009). Reclassification of the members of the genus Tetrathiobacter Ghosh et al. 2005 to the genus Advenella Coenye et al. 2005. Int J Syst Evol Microbiol 59, 1914-1918.

Grossman, M. J., Lee, M. K., Prince, R. C., Minak-Bernero, V., George, G. N. \& Pickering, I. J. (2001). Deep desulphurization of extensively hydrodesulphurized middle distillate oil by Rhodococcus sp. strain ECRD-1. Appl Environ Microbiol 67, 1949-1952.

Gupta, R., Gupta, N. \& Rathi, P. (2004). Bacterial lipases: an overview of production, purification and biochemical properties. Appl Microbiol Biotechnol 64, 763-781.

Hanahan, D. (1983). Studies on transformation of Escherichia coli with plasmids. J Mol Biol 166, 557-580.

Held, K. D. \& Biaglow, J. E. (1994). Mechanisms for the oxygen radical-mediated toxicity of various thiol-containing compounds in cultured mammalian cells. Radiat Res 139, 15-23.
Innis, M. A., Gelfand, D. H., Suinsky, J. J. \& White, T. J. (1990). $P C R$ Protocols: a Guide to Methods and Applications. San Diego, CA: Academic Press.

Jakubowski, H. \& Fersht, A. R. (1981). Alternative pathways of editing noncognate amino acids by aminoacyl-tRNA synthetases. Nucleic Acids Res 9, 3105-3117.

Jakubowski, H. \& Goldman, E. (1992). Editing of errors in selection of amino acids for protein synthesis. Microbiol Rev 56, 412-429.

Jang, L.-S. \& Keng, H.-K. (2006). Development and characterization of $4,4^{\prime}$-dithiodibutyric acid as a monolayer for protein chips. Sens Mater 18, 367-380.

Kalscheuer, R., Arenskötter, M. \& Steinbüchel, A. (1999). Establishment of a gene transfer system for Rhodococcus opacus PD630 based on electroporation and its application for recombinant biosynthesis of poly(3-hydroxyalkanoic acids). Appl Microbiol Biotechnol 52, 508-515.

Kanayama, N. \& Kitano, H. (2000). Interfacial recognition of sugars by boronic acid-carrying self-assembled monolayers. Langmuir 16, $577-$ 583.

Kim, D. Y., Elbanna, K., Thakor, N., Lütke-Eversloh, T. \& Steinbüchel, A. (2005). Poly(3-mercaptopropionate): a non-biodegradable biopolymer? Biomacromolecules 6, 897-901.

Liu, S. J. \& Steinbüchel, A. (2000). A novel genetically engineered pathway for synthesis of poly(hydroxyalkanoic acid) in Escherichia coli. Appl Environ Microbiol 66, 739-743.

Lütke-Eversloh, T. \& Steinbüchel, A. (2003). Novel precursor substrates for polythioesters (PTE) and limits of PTE biosynthesis in Ralstonia eutropha. FEMS Microbiol Lett 221, 191-196.

Lütke-Eversloh, T. \& Steinbüchel, A. (2004). Microbial polythioesters. Macromol Biosci 4, 166-174.

Lütke-Eversloh, T., Bergander, K., Luftmann, H. \& Steinbüchel, A. (2001a). Identification of a new class of biopolymer: bacterial synthesis of sulfur-containing polymer with thioester linkages. Microbiology 147, 11-19.

Lütke-Eversloh, T., Bergander, K., Luftmann, H. \& Steinbüchel, A. (2001b). Biosynthesis of poly(3-hydroxybutyrate-co-3-mercaptobutyrate) as a sulfur analogue to poly(3-hydroxybutyrate) (PHB). Biomacromolecules 2, 1061-1065.

Lütke-Eversloh, T., Kawada, J., Marchessault, R. H. \& Steinbüchel, A. (2002a). Characterization of biological polythioesters: physical properties of novel copolymers synthesized by Ralstonia eutropha. Biomacromolecules 3, 159-166.

Lütke-Eversloh, T., Fischer, A., Remminghorst, U., Kawada, J., Marchessault, R. H., Bögershausen, A., Kalwei, M., Eckert, H., Reichelt, R. \& other authors (2002b). Biosynthesis of novel thermoplastic polythioesters by engineered Escherichia coli. Nat Mater 1, 236-240.

Marmur, J. (1961). A procedure for the isolation of deoxyribonucleic acid from microorganisms. J Mol Biol 3, 208-218.

McLeod, M. P., Warren, R. L., Hsiao, W. W., Araki, N., Myhre, M., Fernandes, C., Miyazawa, D., Wong, W., Lillquist, A. L. \& other authors (2006). The complete genome of Rhodococcus sp. RHA1 provides insights into a catabolic powerhouse. Proc Natl Acad Sci U S A 103, 15582-15587.

Nagy, I., Schoofs, G., Vanderleyen, J. \& De Mot, R. (1997). Transposition of the IS21-related element IS1415 in Rhodococcus erythropolis. J Bacteriol 179, 4635-4638.

Pedrós-Alio, C., Mas, J. \& Guerrero, R. (1985). The influence of poly$\beta$-hydroxybutyrate accumulation on cell volume and buoyant density in Alcaligenes eutrophus. Arch Microbiol 143, 178-184.

Sallam, K. I., Mitani, Y. \& Tamura, T. (2006). Construction of random transposition mutagenesis system in Rhodococcus erythropolis using IS1415. J Biotechnol 121, 13-22. 
Sallam, K. I., Tamura, N. \& Tamura, T. (2007). A multipurpose transposon-based vector system mediates protein expression in Rhodococcus erythropolis. Gene 386, 173-182.

Sambrook, J., Fritsch, E. F. \& Maniatis, T. (1989). Molecular Cloning: a Laboratory Manual, 2nd edn. Cold Spring Harbor, NY: Cold Spring Harbor Laboratory.

Sanger, F., Nicklen, S. \& Coulson, A. R. (1977). DNA sequencing with chain-terminating inhibitors. Proc Natl Acad Sci U S A 74, 5463-5467.

Saxena, R. S. \& Gupta, A. (1984). Electrochemical studies on the composition, stability constants and thermodynamics of $\mathrm{Ti}$ (I) complexes with dithiodipropionic acid. Monatsh Chem 115, 12931298.

Schlegel, H. G., Gottschalk, G. \& von Bartha, R. (1961a). Formation and utilization of poly- $\beta$-hydroxybutyric acid by Knallgas bacteria (Hydrogenomonas). Nature 191, 463-465.

Schlegel, H. G., Kaltwasser, H. \& Gottschalk, G. (1961b). Ein Submersverfahren zur Kultur wasserstoffoxidierender Bakterien: Wachstumsphysiologische Untersuchungen. Arch Mikrobiol 38, 209222 (in German).

Scott, G. (1968). Mechanisms of antioxidant action: esters of thiodipropionic acid. Chem Commun 24, 1572-1574.

Simon, R., Priefer, U. \& Pühler, A. (1983). A broad host range mobilization system for in vivo genetic engineering: transposon mutagenesis in Gram-negative bacteria. Biotechnology (N Y) 1, 784791.

Steinbüchel, A. (2001). Perspectives for biotechnological production and utilization of biopolymers: metabolic engineering of polyhydroxyalkanoate biosynthesis pathways as a successful example. Macromol Biosci 1, 1-24.

Thakor, N., Lütke-Eversloh, T. \& Steinbüchel, A. (2005). Application of the BPEC pathway for large-scale biotechnological production of poly(3-mercaptopropionate) by recombinant Escherichia coli, including a novel in situ isolation method. Appl Environ Microbiol 71, 835841.
Timm, A., Byrom, D. \& Steinbüchel, A. (1990). Formation of blends of various poly(3-hydroxyalkanoic acids) by a recombinant strain of Pseudomonas oleovorans. Appl Microbiol Biotechnol 33, 296-301.

Toups, M., Wübbeler, J. H. \& Steinbüchel, A. (2009). Microbial utilization of the industrial wastewater pollutants 2-ethylhexylthioglycolic acid and iso-octylthioglycolic acid by aerobic Gram-negative bacteria. Biodegradation, doi:10.1007/s10532-009-9302-y.

Tsutsumi, H., Okada, S. \& Oishi, T. (1998). A potentially biodegradable polyamide containing disulfide bonds as a positive material for secondary batteries. Electrochim Acta 43, 427-429.

Tuan, Y.-H. \& Phillips, R. D. (1997). Optimized determination of cystine/cysteine and acid-stable amino acids from a single hydrolysate of casein- and sorghum-based diet and digesta samples. J Agric Food Chem 45, 3535-3540.

Williams, C. H., Jr (1992). Lipoamide dehydrogenase, glutathione reductase, thioredoxin reductase, and mercuric ion reductase-a family of flavoenzyme transhydrogenases. In Chemistry and Biochemistry of Flavoenzymes, vol. III, pp. 121-211. Edited by F. Muller. Boca Raton, FL: CRC Press.

Wübbeler, J. H., Lütke-Eversloh, T., Vandamme, P., Van Trappen, S. \& Steinbüchel, A. (2006). Tetrathiobacter mimigardefordensis sp. nov., isolated from compost, a betaproteobacterium capable of utilizing the organic disulfide 3,3' -dithiodipropionic acid. Int J Syst Evol Microbiol 56, 1305-1310.

Wübbeler, J. H., Bruland, N., Kretschmer, K. \& Steinbüchel, A. (2008). A novel pathway for the catabolism of the organic sulfur compound 3,3'-dithiodipropionic acid via 3-mercaptopropionic acid and 3-sulfinopropionic acid to propionyl-CoA by the aerobic bacterium Tetrathiobacter mimigardefordensis strain DPN7. Appl Environ Microbiol 74, 4028-4035.

Yamada, H. \& Kobayahashi, M. (1996). Nitrile hydratase and its application to industrial production of acrylamide. Biosci Biotechnol Biochem 60, 1391-1400.

Edited by: H. L. Drake 